\title{
Some fixed point theorems for
}

\section{$(\psi-\phi)$-almost weak contractions in $S$-metric spaces solving conformable differential equations}

\author{
Hemant Kumar Nashine ${ }^{1 *}$, G. S. Saluja² and Rabha W. Ibrahim ${ }^{1,3}$
}

\section{"Correspondence:}

hemantkumarnashine@tdtu.edu.vn

${ }^{1}$ Faculty of Mathematics and

Statistics, Ton Duc Thang University,

Ho Chi Minh City, Vietnam

Full list of author information is

available at the end of the article

\begin{abstract}
The aim of this paper is to establish some fixed point and common fixed point theorems for $(\psi-\phi)$-almost weak contractions in complete $S$-metric spaces, followed by some supportive examples. Our results extend and generalize several results existing in the literature. We employ the outcomes of the fixed point theorems to establish the existence and uniqueness of a solution for a class of conformable differential equations which is a new branch of fractional calculus.
\end{abstract}

MSC: Primary $47 \mathrm{H} 10$; secondary $54 \mathrm{H} 25$

Keywords: Fixed point; Common fixed point; $(\psi-\phi)$-almost weak contraction; S-metric space

\section{Springer}

\section{Introduction and preliminaries}

Fixed point theory is one of the noteworthy and stimulating themes of nonlinear functional analysis that blends topology, analysis, and applied mathematics. By the fixed-point technique, a controllability problem is converted to a fixed-point problem for an applicable nonlinear operator in a function space. An important part of this approach is to guarantee the solvability of an invariant subset for this operator. Through the Banach fixed point theorem, one can get a unique solution of any nonlinear equation if we convert it into operator form, and it is a contraction operator in a complete metric space.

In 1997, Alber and Delabrier introduced in [1] the concept of $\phi$-weak contraction. They proved the existence of fixed points for single-valued maps satisfying weak contraction condition in Hilbert spaces. Rhoades [5] has shown that the result of Alber and Delabrieer is also valid in complete metric spaces. The weak contraction was defined as follows.

Definition 1.1 ([1]) A mapping $\mathcal{T}: X \rightarrow X$ is called a $\phi$-weak contraction if there exists a continuous and nondecreasing function $\phi:[0, \infty) \rightarrow[0, \infty)$ such that $\phi$ is positive on

(c) The Author(s) 2020. This article is licensed under a Creative Commons Attribution 4.0 International License, which permits use sharing, adaptation, distribution and reproduction in any medium or format, as long as you give appropriate credit to the original author(s) and the source, provide a link to the Creative Commons licence, and indicate if changes were made. The images or other third party material in this article are included in the article's Creative Commons licence, unless indicated otherwise in a credit line to the material. If material is not included in the article's Creative Commons licence and your intended use is not permitted by statutory regulation or exceeds the permitted use, you will need to obtain permission directly from the copyright holder. To view a copy of this licence, visit http://creativecommons.org/licenses/by/4.0/. 
$(0,+\infty)$ and $\phi(0)=0$, and, for all $x, y \in X$,

$$
d(\mathcal{T}(x), \mathcal{T}(y)) \leq d(x, y)-\phi(d(x, y))
$$

In fact, Banach contraction appears to be a special case of weak contraction by taking $\phi(t)=(1-\alpha) t$ for $0 \leq \alpha<1$. In this connection Rhoades [5] proved the following very interesting fixed point theorem.

Theorem 1.2 Let $(X, d)$ be a complete metric space and let $\mathcal{T}: X \rightarrow X$ be a $\phi$-weak contraction on $X$. Then $\mathcal{T}$ has a unique fixed point.

Dutta and Choudhury introduced in 2008 [4] a new generalization of contraction principle and proved the following theorem.

Theorem 1.3 ([4]) Let $(X, d)$ be a complete metric space and let $\mathcal{T}: X \rightarrow X$ be a selfmapping satisfying the inequality

$$
\psi(d(\mathcal{T}(x), \mathcal{T}(y))) \leq \psi(d(x, y))-\phi(d(x, y))
$$

for all $x, y \in X$, where $\psi, \phi:[0, \infty) \rightarrow[0, \infty)$ are both continuous and nondecreasing functions with $\psi(t)=0=\phi(t)$ if and only if $t=0$. Then $\mathcal{T}$ has a unique fixed point.

Remark 1.4 (i) If we take $\psi(t)=t$ for all $t \geq 0$, then (1.2) reduces to (1.1).

(ii) If we take $\psi(t)=t$ for all $t \geq 0$ and $\phi(t)=(1-\alpha) t$ where $0<\alpha<1$, then (1.2) reduces to contraction condition.

In 2009, Doric [3] generalized Theorem 1.3 for a pair of maps as follows.

Theorem 1.5 ([3]) Let $(X, d)$ be a complete metric space and let $\mathcal{T}, f: X \rightarrow X$ be two selfmappings satisfying the inequality

$$
\psi(d(\mathcal{T}(x), f(y))) \leq \psi(M(x, y))-\phi(M(x, y))
$$

for any $x, y \in X$, where $M$ is given by

$$
M(x, y)=\max \left\{d(x, y), d(x, \mathcal{T} x), d(y, f y), \frac{1}{2}[d(x, f y)+d(y, \mathcal{T} x)]\right\}
$$

and

(a) $\psi:[0, \infty) \rightarrow[0, \infty)$ is a continuous non-decreasing function with $\psi(t)=0$ if and only if $t=0$,

(b) $\phi:[0, \infty) \rightarrow[0, \infty)$ is a lower semi-continuous function with $\phi(t)=0$ if and only if $t=0$.

Then there exists a unique point $u \in X$ such that $u=\mathcal{T} u=f u$.

Recently, Sedghi et al. [6] have introduced the notion of an $S$-metric space as a generalization of $G$-metric spaces and $D^{*}$-metric spaces. 
Definition 1.6 ([6]) Let $X$ be a nonempty set and $S: X^{3} \rightarrow[0, \infty)$ be a function satisfying the following conditions for all $x, y, z, t \in X$ :

$\left(S M_{1}\right) S(x, y, z)=0$ if and only if $x=y=z$;

$\left(S M_{2}\right) S(x, y, z) \leq S(x, x, t)+S(y, y, t)+S(z, z, t)$.

Then the function $S$ is called an $S$-metric on $X$ and the pair $(X, S)$ is called an $S$-metric space, or simply SMS.

Example 1.7 ([6,7]) Let $X=\mathbb{R}^{n}$ and $\|\cdot\|$ be a norm on $X$; then

1. $S(x, y, z)=\|y+z-2 x\|+\|y-z\|$ is an $S$-metric on $X$.

2. $S(x, y, z)=\|x-z\|+\|y-z\|$ is an $S$-metric on $X$.

In the case when $X=\mathbb{R}, S(x, y, z)=|x-z|+|y-z|$ is called the usual $S$-metric on $X$.

Lemma 1.8 ([6, Lemma 2.5]) In an S-metric space, we have $S(x, x, y)=S(y, y, x)$ for all $x, y \in$ $X$.

Definition 1.9 ([6]) Let $(X, S)$ be an $S$-metric space.

(1) A sequence $\left\{x_{n}\right\}$ in $X$ converges to $x \in X$ if $S\left(x_{n}, x_{n}, x\right) \rightarrow 0$ as $n \rightarrow \infty$, that is, for each $\varepsilon>0$, there exists $n_{0} \in \mathbb{N}$ such that for all $n \geq n_{0}$ we have $S\left(x_{n}, x_{n}, x\right)<\varepsilon$. We denote this by $\lim _{n \rightarrow \infty} x_{n}=x$ or $x_{n} \rightarrow x$ as $n \rightarrow \infty$.

(2) A sequence $\left\{x_{n}\right\}$ in $X$ is called a Cauchy sequence if $S\left(x_{n}, x_{n}, x_{m}\right) \rightarrow 0$ as $n, m \rightarrow \infty$, that is, for each $\varepsilon>0$, there exists $n_{0} \in \mathbb{N}$ such that for all $n, m \geq n_{0}$ we have $S\left(x_{n}, x_{n}, x_{m}\right)<\varepsilon$.

(3) The $S$-metric space $(X, S)$ is called complete if every Cauchy sequence is a convergent sequence.

Lemma 1.10 ([6, Lemma 2.12]) Let $(X, S)$ be an S-metric space. If $x_{n} \rightarrow x$ and $y_{n} \rightarrow y$ as $n \rightarrow \infty$ then $S\left(x_{n}, x_{n}, y_{n}\right) \rightarrow S(x, x, y)$ as $n \rightarrow \infty$.

Definition 1.11 Let $T$ be a self mapping on an $S$-metric space $(X, S)$. Then $\mathcal{T}$ is said to be continuous at $x \in X$ if for any sequence $\left\{x_{n}\right\}$ in $X, x_{n} \rightarrow x$ implies that $\mathcal{T} x_{n} \rightarrow \mathcal{T} x$ as $n \rightarrow \infty$

Very recently, Babu et al. [2] introduced the concept of $(\psi-\phi)$-almost weak contraction in a G-metric space and derived a basic fixed point result.

Definition 1.12 Let $(X, G)$ be a $G$-metric space and let $\mathcal{T}$ be a self-map on $X$. If there exist two continuous and nondecreasing functions $\psi, \phi:[0, \infty) \rightarrow[0, \infty)$ with $\psi(t), \phi(t)>0$ for $t>0, \psi(0)=\phi(0)=0$ and $L_{1} \geq 0$ such that

$$
\psi(G(\mathcal{T} x, \mathcal{T} y, \mathcal{T} z)) \leq \psi(G(x, y, z))-\phi(G(x, y, z))+L_{1} \theta(x, y, z)
$$

for all $x, y, z \in X$, where

$$
\theta(x, y, z)=\min \{G(\mathcal{T} x, x, x), G(\mathcal{T} x, y, y), G(\mathcal{T} x, z, z), G(\mathcal{T} x, y, z)\}
$$

then $\mathcal{T}$ is called a $(\psi-\phi)$-almost weakly contractive map on $X$. 
The purpose of this paper is to first extend the notion of a $(\psi-\phi)$-almost weak contraction to $S$-metric spaces and establish some fixed point and common fixed point results. We give several illustrations to justify the results. Moreover, we utilize this condition to check the solvability of a class of conformable differential equations (a new branch of fractional differential equations).

\section{Main results}

First we introduce the notion of a $(\psi-\phi)$-almost weak contraction in the setting of $S$ metric spaces.

Definition 2.1 Let $(X, S)$ be an $S$-metric space. A map $\mathcal{T}: X \rightarrow X$ is called $(\psi-\phi)$-almost weakly contractive if it satisfies the inequality

$$
\psi(S(\mathcal{T} x, \mathcal{T} y, \mathcal{T} z)) \leq \psi(S(x, y, z))-\phi(S(x, y, z))+L_{1} \theta(x, y, z)
$$

for all $x, y, z \in X, L_{1} \geq 0$, where

$$
\theta(x, y, z)=\min \{S(x, x, \mathcal{T} x), S(y, y, \mathcal{T} x), S(z, z, \mathcal{T} x), S(x, x, \mathcal{T} z)\}
$$

and where $\psi, \phi:[0, \infty) \rightarrow[0, \infty)$ are two continuous and nondecreasing functions with $\psi(t), \phi(t)>0$ for $t>0$ and $\psi(t)=\phi(t)=0$ if and only if $t=0$.

Theorem 2.2 Let $(X, S)$ be a complete $S$-metric space and $\mathcal{T}: X \rightarrow X$ be a $(\psi-\phi)$-almost weakly contractive mapping. Then $\mathcal{T}$ has a unique fixed point in $X$.

Proof We start with any $x_{0} \in X$ and define a sequence $\left\{x_{n}\right\}$ by

$$
x_{n}=\mathcal{T} x_{n-1}, \quad n=1,2, \ldots
$$

If $x_{n+1}=x_{n}$ for some $n \in \mathbb{N}$, then trivially $x_{n} \in \operatorname{Fix}(\mathcal{T})$. Suppose $x_{n+1} \neq x_{n}$ for all $n \in \mathbb{N}$. Using (2.1),

$$
\begin{aligned}
& \psi\left(S\left(x_{n+1}, x_{n+1}, x_{n}\right)\right) \\
& \quad=\psi\left(S\left(\mathcal{T} x_{n}, \mathcal{T} x_{n}, \mathcal{T} x_{n-1}\right)\right) \\
& \quad \leq \psi\left(S\left(x_{n}, x_{n}, x_{n-1}\right)\right)-\phi\left(S\left(x_{n}, x_{n}, x_{n-1}\right)\right)+L_{1} \theta\left(x_{n}, x_{n}, x_{n-1}\right),
\end{aligned}
$$

where

$$
\begin{aligned}
& \theta\left(x_{n}, x_{n}, x_{n-1}\right) \\
&= \min \left\{S\left(x_{n}, x_{n}, \mathcal{T} x_{n}\right), S\left(x_{n}, x_{n}, \mathcal{T} x_{n}\right), S\left(x_{n-1}, x_{n-1}, \mathcal{T} x_{n}\right),\right. \\
&\left.S\left(x_{n}, x_{n}, \mathcal{T} x_{n-1}\right)\right\} \\
&= \min \left\{S\left(x_{n}, x_{n}, x_{n+1}\right), S\left(x_{n}, x_{n}, x_{n+1}\right), S\left(x_{n-1}, x_{n-1}, x_{n+1}\right),\right. \\
&\left.S\left(x_{n}, x_{n}, x_{n}\right)\right\} \\
&= \min \left\{S\left(x_{n}, x_{n}, x_{n+1}\right), S\left(x_{n}, x_{n}, x_{n+1}\right), S\left(x_{n-1}, x_{n-1}, x_{n+1}\right), 0\right\}
\end{aligned}
$$


$=0$.

Therefore,

$$
\begin{aligned}
\psi\left(S\left(x_{n+1}, x_{n+1}, x_{n}\right)\right) & =\psi\left(S\left(\mathcal{T} x_{n}, \mathcal{T} x_{n}, \mathcal{T} x_{n-1}\right)\right) \\
& \leq \psi\left(S\left(x_{n}, x_{n}, x_{n-1}\right)\right)-\phi\left(S\left(x_{n}, x_{n}, x_{n-1}\right)\right)
\end{aligned}
$$

that is,

$$
\psi\left(S\left(x_{n+1}, x_{n+1}, x_{n}\right)\right) \leq \psi\left(S\left(x_{n}, x_{n}, x_{n-1}\right)\right)
$$

which implies that

$$
S\left(x_{n+1}, x_{n+1}, x_{n}\right) \leq S\left(x_{n}, x_{n}, x_{n-1}\right), \quad \text { for } n=1,2, \ldots
$$

Therefore $\left\{S\left(x_{n+1}, x_{n+1}, x_{n}\right)\right\}$ is a decreasing sequence of nonnegative reals and hence there exists $d \geq 0$ such that

$$
\lim _{n \rightarrow \infty} S\left(x_{n+1}, x_{n+1}, x_{n}\right)=\gamma \geq 0 .
$$

Letting $n \rightarrow \infty$ in (2.5) gives

$$
\psi(\gamma) \leq \psi(\gamma)-\phi(\gamma)
$$

which is a contradiction unless $\gamma=0$. Hence,

$$
\lim _{n \rightarrow \infty} S\left(x_{n+1}, x_{n+1}, x_{n}\right)=0 .
$$

Next we prove that $\left\{x_{n}\right\}$ is a Cauchy sequence. If not, then there exists an $\varepsilon>0$ for which we can find subsequences $\left\{x_{m(k)}\right\}$ and $\left\{x_{n(k)}\right\}$ of $\left\{x_{n}\right\}$ and increasing sequences of integers $\{m(k)\}$ and $\{n(k)\}$ such that $n(k)$ is the smallest index for which

$$
\begin{aligned}
& n(k)>m(k)>k, \\
& S\left(x_{m(k)}, x_{m(k)}, x_{n(k)}\right) \geq \varepsilon .
\end{aligned}
$$

Then, we have

$$
S\left(x_{m(k)}, x_{m(k)}, x_{n(k)-1}\right)<\varepsilon .
$$

Now using (2.12), $\left(S M_{2}\right)$, and Lemma 1.8, we have

$$
\begin{aligned}
\varepsilon & \leq S\left(x_{m(k)}, x_{m(k)}, x_{n(k)}\right)=S\left(x_{n(k)}, x_{n(k)}, x_{m(k)}\right) \\
& \leq 2 S\left(x_{n(k)}, x_{n(k)}, x_{n(k)-1}\right)+S\left(x_{m(k)}, x_{m(k)}, x_{n(k)-1}\right) \\
& \leq \varepsilon+2 S\left(x_{n(k)}, x_{n(k)}, x_{n(k)-1}\right) \quad(\text { by }(2.13)) .
\end{aligned}
$$


Letting $k \rightarrow \infty$ in (2.14) and using (2.10), we get

$$
\lim _{k \rightarrow \infty} S\left(x_{m(k)}, x_{m(k)}, x_{n(k)}\right)=\varepsilon
$$

From $\left(\mathrm{SM}_{2}\right)$ and Lemma 1.8,

$$
\begin{aligned}
& S\left(x_{m(k)}, x_{m(k)}, x_{n(k)}\right) \\
& \leq 2 S\left(x_{m(k)}, x_{m(k)}, x_{m(k)-1}\right)+S\left(x_{n(k)}, x_{n(k)}, x_{m(k)-1}\right) \\
& \leq 2 S\left(x_{m(k)}, x_{m(k)}, x_{m(k)-1}\right)+2 S\left(x_{n(k)}, x_{n(k)}, x_{n(k)-1}\right) \\
& \quad S\left(x_{m(k)-1}, x_{m(k)-1}, x_{n(k)-1}\right)
\end{aligned}
$$

and

$$
\begin{aligned}
& S\left(x_{m(k)-1}, x_{m(k)-1}, x_{n(k)-1}\right) \\
& \quad \leq 2 S\left(x_{m(k)-1}, x_{m(k)-1}, x_{m(k)}\right)+S\left(x_{n(k)-1}, x_{n(k)-1}, x_{m(k)}\right) \\
& \quad=2 S\left(x_{m(k)}, x_{m(k)}, x_{m(k)-1}\right)+S\left(x_{m(k)}, x_{m(k)}, x_{n(k)-1}\right) .
\end{aligned}
$$

Letting $k \rightarrow \infty$ in (2.17) and using (2.10), (2.13), (2.15), and (2.16), we get

$$
\lim _{k \rightarrow \infty} S\left(x_{m(k)-1}, x_{m(k)-1}, x_{n(k)-1}\right)=\varepsilon
$$

Setting $x=x_{m(k)-1}, y=x_{m(k)-1}$ and $z=x_{n(k)-1}$ in (2.1), we obtain

$$
\begin{aligned}
\psi(\varepsilon) \leq & \psi\left(S\left(x_{m(k)}, x_{m(k)}, x_{n(k)}\right)\right)=\psi\left(S\left(\mathcal{T} x_{m(k)-1}, \mathcal{T} x_{m(k)-1}, \mathcal{T} x_{n(k)-1}\right)\right) \\
\leq & \psi\left(S\left(x_{m(k)-1}, x_{m(k)-1}, x_{n(k)-1}\right)\right)-\phi\left(S\left(x_{m(k)-1}, x_{m(k)-1}, x_{n(k)-1}\right)\right) \\
& +L_{1} \theta\left(x_{m(k)-1}, x_{m(k)-1}, x_{n(k)-1}\right) .
\end{aligned}
$$

Letting $k \rightarrow \infty$ in (2.19) and using (2.10), (2.15), and (2.18), we get

$$
\psi(\varepsilon) \leq \psi(\varepsilon)-\phi(\varepsilon)
$$

a contradiction since $\varepsilon>0$. This shows that $\left\{x_{n}\right\}$ is a Cauchy sequence in the complete $S$ metric space $(X, S)$. So, suppose $x_{n} \rightarrow u$ as $n \rightarrow \infty$. Now we prove that $u \in \operatorname{Fix}(\mathcal{T})$. Using (2.1) with $x=x_{n}, y=x_{n}$, and $z=u$ gives

$$
\begin{aligned}
\psi\left(S\left(x_{n+1}, x_{n+1}, \mathcal{T} u\right)\right) & =\psi\left(S\left(\mathcal{T} x_{n}, \mathcal{T} x_{n}, \mathcal{T} u\right)\right) \\
& \leq \psi\left(S\left(x_{n}, x_{n}, u\right)\right)-\phi\left(S\left(x_{n}, x_{n}, u\right)\right)+L_{1} \theta\left(x_{n}, x_{n}, u\right)
\end{aligned}
$$

Letting $n \rightarrow \infty$ in (2.20), we obtain

$$
\psi(S(u, u, \mathcal{T} u)) \leq \psi(S(u, u, u))-\phi(S(u, u, u))=0
$$


so that $S(u, u, \mathcal{T} u)=0$. Hence $u=\mathcal{T} u$. This shows that $u \in \operatorname{Fix}(\mathcal{T})$. To prove the uniqueness of $u$, let $v$ be a fixed point of $\mathcal{T}$ with $v \neq u$. Then from (2.1), we have

$$
\begin{aligned}
\psi(S(u, u, v)) & =\psi(S(\mathcal{T} u, \mathcal{T} u, \mathcal{T} v)) \\
& \leq \psi(S(u, u, v))-\phi(S(u, u, v))+L_{1} \theta(u, u, v) \\
& \leq \psi(S(u, u, v))-\phi(S(u, u, v))
\end{aligned}
$$

a contradiction unless $S(u, u, v)=0$. Hence $u=v$. This shows that the fixed point of $\mathcal{T}$ is unique.

If $L_{1}=0$ in Theorem 2.2, then we obtain the following result.

Corollary 2.3 Let $(X, S)$ be a complete $S$-metric space and $\mathcal{T}: X \rightarrow X$ be a self-mapping satisfying the inequality

$$
\psi(S(\mathcal{T} x, \mathcal{T} y, \mathcal{T} z)) \leq \psi(S(x, y, z))-\phi(S(x, y, z))
$$

for all $x, y, z \in X$, and where $\psi$ and $\phi$ are functions defined as in Theorem 2.2. Then $\mathcal{T}$ has a unique fixed point in $X$.

Remark 2.4 Corollary 2.3 extends Theorem 2.1 of Dutta and Choudhury [4] from a complete metric space to that in the setting of a complete $S$-metric space considered in this paper.

If, in Theorem 2.2, $L_{1}=0$ and $\psi$ is the identity map, then we obtain the following result.

Corollary 2.5 Let $(X, S)$ be a complete $S$-metric space and $T: X \rightarrow X$ be a self-mapping satisfying the inequality

$$
S(\mathcal{T} x, \mathcal{T} y, \mathcal{T} z) \leq S(x, y, z)-\phi(S(x, y, z))
$$

for all $x, y, z \in X$, and where $\phi$ is a continuous and nondecreasing function defined on $[0, \infty)$ with $\phi(t)=0$ if and only if $t=0$. Then $T$ has a unique fixed point in $X$.

Remark 2.6 Corollary 2.5 extends the corresponding result of Rhoades [5] from a complete metric space to that in the setting of a complete $S$-metric space considered in this paper.

\subsection{Illustrations}

Example 2.7 Let $X=[0,1] \cup\{2,3,4, \ldots\}$ and

$$
S(x, y, z)= \begin{cases}|x-y-z| & \text { if } x, y, z \in[0,1], x \neq y \neq z, \\ x+y+z & \text { if at least one of } x \text { or } y \text { or } z \notin[0,1] \text { and } x \neq y \neq z, \\ 0 & \text { if } x=y=z,\end{cases}
$$

for all $x, y, z \in X$. Then $(X, S)$ is a complete $S$-metric space. 
Let $\psi:[0, \infty) \rightarrow[0, \infty)$ be defined as

$$
\psi(t)= \begin{cases}t & \text { if } 0 \leq t \leq 1 \\ t^{2} & \text { if } t>1\end{cases}
$$

and let $\phi:[0, \infty) \rightarrow[0, \infty)$ be defined as

$$
\phi(t)= \begin{cases}t^{2} & \text { if } 0 \leq t \leq 1, \\ \frac{1}{2} & \text { if } t>1\end{cases}
$$

Let $\mathcal{T}: X \rightarrow X$ be defined as

$$
\mathcal{T}(x)= \begin{cases}x-x^{2} & \text { if } 0 \leq x \leq 1 \\ x-1 & \text { if } x \in\{2,3,4, \ldots\}\end{cases}
$$

Without loss of generality, we assume that $x>y>z$ with $x=\frac{3}{4}, y=\frac{1}{2}$, and $z=\frac{1}{4}$ and discuss the following cases.

Case I. If $x \in[0,1]$, then

$$
\begin{aligned}
\psi(S(\mathcal{T} x, \mathcal{T} y, \mathcal{T} z)) & =S(\mathcal{T} x, \mathcal{T} y, \mathcal{T} z) \\
& =S\left(x-x^{2}, y-y^{2}, z-z^{2}\right) \\
& =\left[\left(x-x^{2}\right)-\left(y-y^{2}\right)-\left(z-z^{2}\right)\right] \\
& =\left[(x-y-z)-\left(x^{2}-y^{2}-z^{2}\right)\right] \\
& \leq\left[(x-y-z)-(x-y-z)^{2}\right] \\
& =S(x, y, z)-(S(x, y, z))^{2} \\
& =\psi(S(x, y, z))-\phi(S(x, y, z)) .
\end{aligned}
$$

Case II. If $x \in\{2,3,4, \ldots\}$, then

$$
S(\mathcal{T} x, \mathcal{T} y, \mathcal{T} z)=S\left(x-1, y-1, z-z^{2}\right) \quad \text { if } z \in[0,1]
$$

or

$$
\begin{aligned}
S(\mathcal{T} x, \mathcal{T} y, \mathcal{T} z) & =(x-1)+(y-1)+\left(z-z^{2}\right)=x+y+z-z^{2}-2 \\
& \leq x+y+z-2
\end{aligned}
$$

and

$$
S(\mathcal{T} x, \mathcal{T} y, \mathcal{T} z)=S(x-1, y-1, z-1) \quad \text { if } y, z \in\{2,3,4, \ldots\}
$$

or

$S(\mathcal{T} x, \mathcal{T} y, \mathcal{T} z)=x-1+y-1+z-1=x+y+z-3$ 


$$
\leq x+y+z-2
$$

Consequently,

$$
\begin{aligned}
\psi(S(\mathcal{T} x, \mathcal{T} y, \mathcal{T} z)) & =S(\mathcal{T} x, \mathcal{T} y, \mathcal{T} z)^{2} \\
& \leq(x+y+z-2)^{2} \\
& <(x+y+z-1)(x+y+z+1) \\
& =(x+y+z)^{2}-1<(x+y+z)^{2}-\frac{1}{2} \\
& =(S(x, y, z))^{2}-\phi(S(x, y, z)) \\
& =\psi(S(x, y, z))-\phi(S(x, y, z)) .
\end{aligned}
$$

Case III. If $x=2$, then $y, z \in[0,1], T(x)=1$, and $S(\mathcal{T} x, \mathcal{T} x, \mathcal{T} y)=1+\left(y-y^{2}\right)+\left(z-z^{2}\right) \leq 2$. So, we have $\psi(S(\mathcal{T} x, \mathcal{T} y, \mathcal{T} z)) \leq \psi(2)=4$. Again $S(x, y, z)=2+y+z$. So,

$$
\begin{aligned}
\psi(S(x, y, z))-\phi(S(x, y, z)) & =(2+y+z)^{2}-\phi\left((2+y+z)^{2}\right) \\
& =(2+y+z)^{2}-\frac{1}{2} \\
& =\frac{7}{2}+y^{2}+z^{2}+2 y z+4 y+4 z>4 \\
& =\psi(S(\mathcal{T} x, \mathcal{T} y, \mathcal{T} z)) .
\end{aligned}
$$

Considering all the above cases, we conclude that the inequality used in Corollary 2.3 remains valid for $\psi, \phi$, and $\mathcal{T}$ constructed in above example and, consequently by an application of Corollary 2.3, $\mathcal{T}$ has a unique fixed point which is 0.

Example 2.8 Let $X=[0,1]$ and let $S$ be the $S$-metric defined by $S(x, y, z)=|x-z|+|y-z|$ for all $x, y, z \in X$. Then $(X, S)$ is a complete $S$-metric space. Let $\mathcal{T}: X \rightarrow X$ be a mapping defined as $\mathcal{T}(x)=x-\frac{x^{2}}{2}$ and $\phi(t)=\frac{t^{2}}{4}$. Without loss of generality, we assume that $x>y>z$. Then for $x=1, y=\frac{1}{2}$, and $z=\frac{1}{4}$, we have

$$
\begin{aligned}
S(\mathcal{T} x, \mathcal{T} y, \mathcal{T} z) & =|T x-T z|+|T y-T z| \\
& =\left|\left(x-\frac{x^{2}}{2}\right)-\left(z-\frac{z^{2}}{2}\right)\right|+\left|\left(y-\frac{y^{2}}{2}\right)-\left(z-\frac{z^{2}}{2}\right)\right| \\
& =\left(x-\frac{x^{2}}{2}\right)-\left(z-\frac{z^{2}}{2}\right)+\left(y-\frac{y^{2}}{2}\right)-\left(z-\frac{z^{2}}{2}\right) \\
& \leq[(x-z)+(y-z)]-\frac{1}{2}\left[(x-z)^{2}+(y-z)^{2}\right] \\
& \leq[(x-z)+(y-z)]-\frac{1}{4}[(x-z)+(y-z)]^{2} \\
& =S(x, y, z)-\phi(S(x, y, z)) .
\end{aligned}
$$

Thus $\mathcal{T}$ satisfies inequality (2.24) so that $\mathcal{T}$ is a weakly contractive map. Thus by Corollary $2.5, \mathcal{T}$ has a unique fixed point 0 in $X$. 
Example 2.9 Let $X=[0,1]$. We define $S: X^{3} \rightarrow \mathbb{R}_{+}$by

$$
S(x, y, z)= \begin{cases}0 & \text { if } x=y=z, \\ \max \{x, y, z\} & \text { if otherwise }\end{cases}
$$

for all $x, y, z \in X$. Then $(X, S)$ is a complete $S$-metric space. Let $\mathcal{T}: X \rightarrow X$ be a mapping defined as $\mathcal{T}(x)=\frac{x^{2}}{2}$ and $\phi(t)=\frac{t^{2}}{4}$. Without loss of generality, we assume that $x>y>z$. Then

$$
\begin{aligned}
& S(\mathcal{T} x, \mathcal{T} y, \mathcal{T} z)=\max \{\mathcal{T} x, \mathcal{T} y, \mathcal{T} z\}=\frac{x^{2}}{2}, \\
& S(x, y, z)=\max \{x, y, z\}=x
\end{aligned}
$$

and

$$
\phi(S(x, y, z))=\frac{x^{2}}{4}
$$

Now, $S(x, y, z)-\phi(S(x, y, z))=x-\frac{x^{2}}{4}$. Therefore $S(\mathcal{T} x, \mathcal{T} y, \mathcal{T} z)=\frac{x^{2}}{2}<x-\frac{x^{2}}{4}=S(x, y, z)-$ $\phi(S(x, y, z))$. Hence $\mathcal{T}$ satisfies inequality (2.23) so that $\mathcal{T}$ is a weakly contractive map. Thus by Corollary 2.3, $\mathcal{T}$ has a unique fixed point and clearly it is 0 in $X$.

Example 2.10 Let $X=[0,1]$. We define $S: X^{3} \rightarrow \mathbb{R}_{+}$by

$$
S(x, y, z)= \begin{cases}0 & \text { if } x=y=z \\ \max \{x, y, z\} & \text { if otherwise }\end{cases}
$$

for all $x, y, z \in X$. Then $(X, S)$ is a complete $S$-metric space. We define $\mathcal{T}: X \rightarrow X$ and $\psi, \phi$ on $\mathbb{R}_{+}$by $\mathcal{T}(x)=\frac{2 x}{3}, \psi(t)=\frac{9}{8} t^{2}$ and $\phi(t)=\frac{1}{8} t^{2}$ for all $x \in X$ and $t \in \mathbb{R}_{+}$.

Without loss of generality, we assume that $x>y>z$. Then choosing $x=1, y=\frac{1}{2}$, and $z=\frac{1}{4}$, we have

$$
\begin{aligned}
& S(\mathcal{T} x, \mathcal{T} y, \mathcal{T} z)=\max \left\{\frac{2 x}{3}, \frac{2 y}{3}, \frac{2 z}{3}\right\}=\frac{2 x}{3}, \\
& S(x, y, z)=\max \{x, y, z\}=x, \\
& S(y, y, \mathcal{T} y)=\max \left\{y, y, \frac{2 y}{3}\right\}=y, \\
& S(z, z, \mathcal{T} z)=\max \left\{z, z, \frac{2 z}{3}\right\}=z, \\
& S(x, x, \mathcal{T} z)=\max \left\{x, x, \frac{2 z}{3}\right\}=x, \\
& S(z, z, \mathcal{T} x)=\max \left\{z, z, \frac{2 x}{3}\right\}=\frac{2 x}{3},
\end{aligned}
$$

and

$$
\Theta(x, y, z)=\max \left\{x, y, z, \frac{1}{2}\left(x+\frac{2 x}{3}\right)\right\}=x .
$$


Now, we consider

$$
\begin{aligned}
\psi(S(\mathcal{T} x, \mathcal{T} y, \mathcal{T} z)) & =\frac{x^{2}}{2} \leq x^{2}=[\Theta(x, y, z)]^{2} \\
& =\frac{9}{8}[\Theta(x, y, z)]^{2}-\frac{1}{8}[\Theta(x, y, z)]^{2} \\
& =\psi(\Theta(x, y, z))-\phi(\Theta(x, y, z)),
\end{aligned}
$$

that is,

$$
\psi(S(\mathcal{T} x, \mathcal{T} y, \mathcal{T} z)) \leq \psi(\Theta(x, y, z))-\phi(\Theta(x, y, z))
$$

Thus inequality (2.23) of Corollary 2.3 holds, and 0 is the unique fixed point of $\mathcal{T}$.

Example 2.11 Let $X=[0,1]$. We define $S: X^{3} \rightarrow \mathbb{R}_{+}$by

$$
S(x, y, z)= \begin{cases}0 & \text { if } x=y=z \\ \max \{x, y, z\} & \text { if otherwise }\end{cases}
$$

for all $x, y, z \in X$. Then $(X, S)$ is a complete $S$-metric space. Let $\mathcal{T}: X \rightarrow X$ be a mapping defined as

$$
\mathcal{T}(x)= \begin{cases}\frac{1}{2} & \text { if } x=0 \\ 2 x & \text { if } 0<x<\frac{1}{2} \\ 1 & \text { if } \frac{1}{2} \leq x \leq 1\end{cases}
$$

We define $\psi$ and $\phi$ on $\mathbb{R}_{+}$by $\psi(t)=\frac{t^{2}}{3}$ and $\phi(t)=\frac{t^{2}}{2}$. Then, if we take $x=y=z=0$ and $L_{1}=1$, we have

$$
\begin{aligned}
& S(\mathcal{T} x, \mathcal{T} y, \mathcal{T} z)=S(\mathcal{T}(0), \mathcal{T}(0), \mathcal{T}(0))=S\left(\frac{1}{2}, \frac{1}{2}, \frac{1}{2}\right)=\max \left\{\frac{1}{2}, \frac{1}{2}, \frac{1}{2}\right\}=\frac{1}{2} \\
& S(x, y, z)=\max \{x, y, z\}=\max \{0,0,0\}=0 \\
& S(x, x, \mathcal{T} x)=\max \left\{0,0, \frac{1}{2}\right\}=\frac{1}{2} \\
& S(y, y, \mathcal{T} y)=\max \left\{0,0, \frac{1}{2}\right\}=\frac{1}{2} \\
& S(z, z, \mathcal{T} x)=\max \left\{0,0, \frac{1}{2}\right\}=\frac{1}{2} \\
& S(x, x, \mathcal{T} z)=\max \left\{0,0, \frac{1}{2}\right\}=\frac{1}{2}
\end{aligned}
$$

and

$$
\theta(x, y, z)=\min \left\{\frac{1}{2}, \frac{1}{2}, \frac{1}{2}, \frac{1}{2}\right\}=\frac{1}{2} .
$$


Now, we have

$$
\begin{aligned}
& \psi(S(\mathcal{T} x, \mathcal{T} y, \mathcal{T} z))=\frac{1}{12}, \\
& \psi(S(x, y, z))=0, \quad \phi(S(x, y, z))=0 .
\end{aligned}
$$

Thus, we have

$$
\begin{aligned}
\psi(S(\mathcal{T} x, \mathcal{T} y, \mathcal{T} z)) & =\frac{1}{12} \leq 0-0+1 \cdot \frac{1}{2}=\frac{1}{2} \\
& =\psi(S(x, y, z))-\phi(S(x, y, z))+L_{1} \theta(x, y, z)
\end{aligned}
$$

that is,

$$
\psi(S(\mathcal{T} x, \mathcal{T} y, \mathcal{T} z)) \leq \psi(S(x, y, z))-\phi(S(x, y, z))+L_{1} \theta(x, y, z)
$$

Hence $\mathcal{T}$ is a $(\psi-\phi)$-almost weak contraction. Thus $\mathcal{T}$ satisfies all the hypothesis of Theorem 2.2 and 1 is the unique fixed point of $\mathcal{T}$.

\section{Common fixed point results}

In this section, we discuss a common fixed point theorem for a pair of maps in the setting of $S$-metric spaces.

Theorem 3.1 Let $(X, S)$ be a complete S-metric space and $F, H: X \rightarrow X$ be two selfmappings satisfying the inequality

$$
\psi(S(F x, F y, H z)) \leq \psi(\Theta(x, y, z))-\phi(\Theta(x, y, z))+L_{1} \theta(x, y, z)
$$

for all $x, y, z \in X, L_{1} \geq 0$, where

$$
\begin{aligned}
\Theta(x, y, z)= & \max \{S(x, y, z), S(y, y, F x), S(z, z, H z), \\
& \left.\frac{1}{2}[S(y, y, H z)+S(z, z, H y)]\right\}, \\
\theta(x, y, z)= & \min \{S(x, x, F x), S(y, y, H y), S(x, x, H y), S(y, y, F z)\},
\end{aligned}
$$

and where $\psi$ and $\phi$ are functions defined as in Theorem 2.2. Then there exists a unique point $u \in X$ such that $u=F u=H u$.

Proof For any $x_{0} \in X$, we construct a sequence $\left\{x_{n}\right\}$ for $n \geq 0$ recursively as

$$
x_{2 n+1}=H x_{2 n}, \quad x_{2 n}=F x_{2 n+1} .
$$

Suppose now that $n$ is an odd number. Putting $x=x_{n}, y=x_{n}$, and $z=x_{n-1}$ in inequality (3.1), we get

$$
\psi\left(S\left(x_{n+1}, x_{n+1}, x_{n}\right)\right)
$$




$$
\begin{aligned}
& =\psi\left(S\left(F x_{n}, F x_{n}, H x_{n-1}\right)\right) \\
& \leq \psi\left(\Theta\left(x_{n}, x_{n}, x_{n-1}\right)\right)-\phi\left(\Theta\left(x_{n}, x_{n}, x_{n-1}\right)\right)+L_{1} \theta\left(x_{n}, x_{n}, x_{n-1}\right),
\end{aligned}
$$

where

$$
\begin{aligned}
\theta & \left(x_{n}, x_{n}, x_{n-1}\right) \\
& =\min \left\{S\left(x_{n}, x_{n}, F x_{n}\right), S\left(x_{n}, x_{n}, H x_{n}\right), S\left(x_{n}, x_{n}, H x_{n}\right), S\left(x_{n}, x_{n}, F x_{n-1}\right)\right\} \\
& =\min \left\{S\left(x_{n}, x_{n}, x_{n+1}\right), S\left(x_{n}, x_{n}, x_{n+1}\right), S\left(x_{n}, x_{n}, x_{n+1}\right), S\left(x_{n}, x_{n}, x_{n}\right)\right\} \\
& =\min \left\{S\left(x_{n}, x_{n}, x_{n+1}\right), S\left(x_{n}, x_{n}, x_{n+1}\right), S\left(x_{n}, x_{n}, x_{n+1}\right), 0\right\} \\
& =0 .
\end{aligned}
$$

Using (3.4) in (3.3), we get

$$
\begin{aligned}
\psi\left(S\left(x_{n+1}, x_{n+1}, x_{n}\right)\right) & =\psi\left(S\left(F x_{n}, F x_{n}, H x_{n-1}\right)\right) \\
& \leq \psi\left(\Theta\left(x_{n}, x_{n}, x_{n-1}\right)\right)-\phi\left(\Theta\left(x_{n}, x_{n}, x_{n-1}\right)\right)
\end{aligned}
$$

which implies

$$
\psi\left(S\left(x_{n+1}, x_{n+1}, x_{n}\right)\right) \leq \psi\left(\Theta\left(x_{n}, x_{n}, x_{n-1}\right)\right) .
$$

Using the properties of $\psi$ and $\phi$ functions in the above inequality, we obtain

$$
S\left(x_{n+1}, x_{n+1}, x_{n}\right) \leq \Theta\left(x_{n}, x_{n}, x_{n-1}\right) .
$$

Now using condition $\left(S M_{2}\right)$ and Lemma 1.8, we have

$$
\begin{aligned}
\Theta\left(x_{n}, x_{n}, x_{n-1}\right)= & \max \left\{S\left(x_{n}, x_{n}, x_{n-1}\right), S\left(x_{n}, x_{n}, F x_{n}\right), S\left(x_{n-1}, x_{n-1}, H x_{n-1}\right),\right. \\
& \left.\frac{1}{2}\left[S\left(x_{n}, x_{n}, H x_{n-1}\right)+S\left(x_{n-1}, x_{n-1}, H x_{n}\right)\right]\right\} \\
= & \max \left\{S\left(x_{n}, x_{n}, x_{n-1}\right), S\left(x_{n}, x_{n}, x_{n+1}\right), S\left(x_{n-1}, x_{n-1}, x_{n}\right),\right. \\
& \left.\frac{1}{2}\left[S\left(x_{n}, x_{n}, x_{n}\right)+S\left(x_{n-1}, x_{n-1}, x_{n+1}\right)\right]\right\} \\
= & \max \left\{S\left(x_{n}, x_{n}, x_{n-1}\right), S\left(x_{n+1}, x_{n+1}, x_{n}\right), S\left(x_{n}, x_{n}, x_{n-1}\right),\right. \\
& \left.\frac{1}{2}\left[S\left(x_{n+1}, x_{n+1}, x_{n-1}\right)\right]\right\} \\
\leq & \max \left\{S\left(x_{n}, x_{n}, x_{n-1}\right), S\left(x_{n+1}, x_{n+1}, x_{n}\right), S\left(x_{n}, x_{n}, x_{n-1}\right),\right. \\
& \left.\frac{1}{2}\left[2 S\left(x_{n+1}, x_{n+1}, x_{n}\right)+S\left(x_{n-1}, x_{n-1}, x_{n}\right)\right]\right\}
\end{aligned}
$$




$$
\begin{aligned}
= & \max \left\{S\left(x_{n}, x_{n}, x_{n-1}\right), S\left(x_{n+1}, x_{n+1}, x_{n}\right), S\left(x_{n}, x_{n}, x_{n-1}\right),\right. \\
& \left.\frac{1}{2}\left[2 S\left(x_{n+1}, x_{n+1}, x_{n}\right)+S\left(x_{n}, x_{n}, x_{n-1}\right)\right]\right\} .
\end{aligned}
$$

If $S\left(x_{n+1}, x_{n+1}, x_{n}\right)>S\left(x_{n}, x_{n}, x_{n-1}\right)$, then $\Theta\left(x_{n}, x_{n}, x_{n-1}\right)=S\left(x_{n+1}, x_{n+1}, x_{n}\right)>0$. This furthermore implies that

$$
\psi\left(S\left(x_{n+1}, x_{n+1}, x_{n}\right)\right) \leq \psi\left(S\left(x_{n+1}, x_{n+1}, x_{n}\right)\right)-\phi\left(S\left(x_{n+1}, x_{n+1}, x_{n}\right)\right)
$$

which is a contraction. So, we have

$$
S\left(x_{n+1}, x_{n+1}, x_{n}\right) \leq \Theta\left(x_{n}, x_{n}, x_{n-1}\right) \leq S\left(x_{n}, x_{n}, x_{n-1}\right)
$$

Similarly, we can obtain the same inequality as above in the case when $n$ is an even number. Therefore the sequence $\left\{S\left(x_{n+1}, x_{n+1}, x_{n}\right)\right\}$ is decreasing and bounded. So there exists $r \geq 0$ such that

$$
\lim _{n \rightarrow \infty} S\left(x_{n+1}, x_{n+1}, x_{n}\right)=\lim _{n \rightarrow \infty} \Theta\left(x_{n}, x_{n}, x_{n-1}\right)=r \geq 0
$$

Letting $n \rightarrow \infty$ in inequality (3.8), we obtain

$$
\psi(r) \leq \psi(r)-\phi(r)
$$

which is a contradiction unless $r=0$. Hence,

$$
\lim _{n \rightarrow \infty} S\left(x_{n+1}, x_{n+1}, x_{n}\right)=0 .
$$

Next we prove that $\left\{x_{n}\right\}$ is a Cauchy sequence. Because of (3.12) it is sufficient to show that $\left\{x_{2 n}\right\}$ is a Cauchy sequence. If not, then there exists $\varepsilon>0$ for which we can find subsequences $\left\{x_{2 m(k)}\right\}$ and $\left\{x_{2 n(k)}\right\}$ of $\left\{x_{2 n}\right\}$ and increasing sequences of integers $\{2 m(k)\}$ and $\{2 n(k)\}$ such that $n(k)$ is the smallest index for which

$$
\begin{aligned}
& n(k)>m(k)>k, \\
& S\left(x_{2 m(k)}, x_{2 m(k)}, x_{2 n(k)}\right) \geq \varepsilon .
\end{aligned}
$$

Then

$$
S\left(x_{2 m(k)}, x_{2 m(k)}, x_{2 n(k)-1}\right)<\varepsilon .
$$

Now using (3.14), $\left(S M_{2}\right)$, and Lemma 1.8, we have

$$
\begin{aligned}
\varepsilon & \leq S\left(x_{2 m(k)}, x_{2 m(k)}, x_{2 n(k)}\right)=S\left(x_{2 n(k)}, x_{2 n(k)}, x_{2 m(k)}\right) \\
& \leq 2 S\left(x_{2 n(k)}, x_{2 n(k)}, x_{2 n(k)-1}\right)+S\left(x_{2 m(k)}, x_{2 m(k)}, x_{2 n(k)-1}\right) \\
& \leq \varepsilon+2 S\left(x_{2 n(k)}, x_{2 n(k)}, x_{2 n(k)-1}\right) \quad(\text { by }(3.15) .
\end{aligned}
$$


Letting $k \rightarrow \infty$ in equation (3.16) and using (3.12), we get

$$
\lim _{k \rightarrow \infty} S\left(x_{2 m(k)}, x_{2 m(k)}, x_{2 n(k)}\right)=\varepsilon
$$

Again, with the help of $\left(S M_{2}\right)$ and Lemma 1.8, we have

$$
\begin{aligned}
& S\left(x_{2 m(k)}, x_{2 m(k)}, x_{2 n(k)}\right) \\
& \quad \leq 2 S\left(x_{2 m(k)}, x_{2 m(k)}, x_{2 m(k)-1}\right)+S\left(x_{2 n(k)}, x_{2 n(k)}, x_{2 m(k)-1}\right) \\
& \quad \leq 2 S\left(x_{2 m(k)}, x_{2 m(k)}, x_{2 m(k)-1}\right)+S\left(x_{2 m(k)-1}, x_{2 m(k)-1}, x_{2 n(k)}\right) .
\end{aligned}
$$

Also, with the help of $\left(S M_{2}\right)$ and Lemma 1.8, we have

$$
\begin{aligned}
& S\left(x_{2 m(k)-1}, x_{2 m(k)-1}, x_{2 n(k)}\right) \\
& \quad \leq 2 S\left(x_{2 m(k)-1}, x_{2 m(k)-1}, x_{2 m(k)}\right)+S\left(x_{2 n(k)}, x_{2 n(k)}, x_{2 m(k)}\right) \\
& \quad=2 S\left(x_{2 m(k)-1}, x_{2 m(k)-1}, x_{2 m(k)}\right)+S\left(x_{2 m(k)}, x_{2 m(k)}, x_{2 n(k)}\right) .
\end{aligned}
$$

Letting $k \rightarrow \infty$ in equation (3.19) and using (3.12), (3.15), (3.17), and (3.18), we get

$$
\lim _{k \rightarrow \infty} S\left(x_{2 m(k)-1}, x_{2 m(k)-1}, x_{2 n(k)}\right)=\varepsilon .
$$

Again, note that with the help of $\left(S M_{2}\right)$ and Lemma 1.8, we have

$$
\begin{aligned}
S\left(x_{2 m(k)}, x_{2 m(k)}, x_{2 n(k)+1}\right) \leq & 2 S\left(x_{2 m(k)}, x_{2 m(k)}, x_{2 m(k)-1}\right) \\
& +S\left(x_{2 n(k)+1}, x_{2 n(k)+1}, x_{2 m(k)-1}\right) \\
\leq & 2 S\left(x_{2 m(k)}, x_{2 m(k)}, x_{2 m(k)-1}\right) \\
& +2 S\left(x_{2 n(k)+1}, x_{2 n(k)+1}, x_{2 n(k)}\right) \\
& +S\left(x_{2 m(k)-1}, x_{2 m(k)-1}, x_{2 n(k)}\right) .
\end{aligned}
$$

Also, with the help of $\left(S M_{2}\right)$ and Lemma 1.8, we have

$$
\begin{aligned}
S\left(x_{2 m(k)-1}, x_{2 m(k)-1}, x_{2 n(k)}\right)= & S\left(x_{2 n(k)}, x_{2 n(k)}, x_{2 m(k)-1}\right) \\
\leq & 2 S\left(x_{2 n(k)}, x_{2 n(k)}, x_{2 n(k)+1}\right) \\
& +S\left(x_{2 m(k)-1}, x_{2 m(k)-1}, x_{2 n(k)+1}\right) \\
= & 2 S\left(x_{2 n(k)+1}, x_{2 n(k)+1}, x_{2 n(k)}\right) \\
& +S\left(x_{2 m(k)-1}, x_{2 m(k)-1}, x_{2 n(k)+1}\right) \\
\leq & 2 S\left(x_{2 n(k)+1}, x_{2 n(k)+1}, x_{2 n(k)}\right) \\
& +2 S\left(x_{2 m(k)-1}, x_{2 m(k)-1}, x_{2 m(k)}\right) \\
& +S\left(x_{2 n(k)+1}, x_{2 n(k)+1}, x_{2 m(k)}\right) \\
= & 2 S\left(x_{2 n(k)+1}, x_{2 n(k)+1}, x_{2 n(k)}\right)
\end{aligned}
$$




$$
\begin{aligned}
& +2 S\left(x_{2 m(k)}, x_{2 m(k)}, x_{2 m(k)-1}\right) \\
& +S\left(x_{2 m(k)}, x_{2 m(k)}, x_{2 n(k)+1}\right) .
\end{aligned}
$$

Letting $k \rightarrow \infty$ in equation (3.22) and using (3.12), (3.20), and (3.21), we get

$$
\lim _{k \rightarrow \infty} S\left(x_{2 m(k)}, x_{2 m(k)}, x_{2 n(k)+1}\right)=\varepsilon
$$

Also, from the definition of $\Theta$ and from equations (3.12), (3.17), and (3.23), we have

$$
\lim _{n \rightarrow \infty} \Theta\left(x_{2 m(k)-1}, x_{2 m(k)-1}, x_{2 n(k)}\right)=\varepsilon
$$

We now consider inequality (3.1). Putting $x=x_{2 m(k)-1}, y=x_{2 m(k)-1}$, and $z=x_{2 n(k)}$, we have

$$
\begin{aligned}
\psi\left(S\left(x_{2 m(k)}, x_{2 m(k)}, x_{2 n(k)+1}\right)\right) \\
=\psi\left(S\left(F x_{2 m(k)-1}, F x_{2 m(k)-1}, H x_{2 n(k)}\right)\right) \\
\leq \psi\left(\Theta\left(x_{2 m(k)-1}, x_{2 m(k)-1}, x_{2 n(k)}\right)\right)-\phi\left(\Theta\left(x_{2 m(k)-1}, x_{2 m(k)-1}, x_{2 n(k)}\right)\right) \\
\quad+L_{1} \theta\left(x_{2 m(k)-1}, x_{2 m(k)-1}, x_{2 n(k)}\right) .
\end{aligned}
$$

Letting $k \rightarrow \infty$ in equation (3.25) and using (3.1), (3.20), (3.23), and (3.24), we get

$$
\psi(\varepsilon) \leq \psi(\varepsilon)-\phi(\varepsilon)<\psi(\varepsilon)
$$

a contradiction. This shows that $\left\{x_{n}\right\}$ is a Cauchy sequence in the complete $S$-metric space $(X, S)$. So, suppose $x_{n} \rightarrow u$ as $n \rightarrow \infty$. Now we prove that $u=F u=H u$. Indeed, suppose $u \neq H u$, then for $S(u, u, H u)>0$, there exists $N_{1} \in \mathbb{N}$ such that for any $n>N_{1}$, we have

$$
\begin{aligned}
& S\left(x_{2 n-1}, x_{2 n-1}, u\right)<\frac{1}{4} S(u, u, H u), \\
& S\left(x_{2 n}, x_{2 n}, x_{2 n-1}\right)<\frac{1}{4} S(u, u, H u), \\
& S\left(x_{2 n}, x_{2 n}, u\right)<\frac{1}{4} S(u, u, H u) .
\end{aligned}
$$

Now, putting $x=x_{2 n-1}, y=x_{2 n-1}$, and $z=u$ in equation (3.1), we obtain

$$
\begin{aligned}
& \psi\left(S\left(x_{2 n}, x_{2 n}, H u\right)\right) \\
& \quad=\psi\left(S\left(F x_{2 n-1}, F x_{2 n-1}, H u\right)\right) \\
& \quad \leq \psi\left(\Theta\left(x_{2 n-1}, x_{2 n-1}, u\right)\right)-\phi\left(\Theta\left(x_{2 n-1}, x_{2 n-1}, u\right)\right)+L_{1} \theta\left(x_{2 n-1}, x_{2 n-1}, u\right)
\end{aligned}
$$

where

$$
\begin{aligned}
& \Theta\left(x_{2 n-1}, x_{2 n-1}, u\right) \\
& \quad=\max \left\{S\left(x_{2 n-1}, x_{2 n-1}, u\right), S\left(x_{2 n-1}, x_{2 n-1}, F x_{2 n-1}\right), S(u, u, H u),\right.
\end{aligned}
$$




$$
\begin{aligned}
& \left.\frac{1}{2}\left[S\left(x_{2 n-1}, x_{2 n-1}, H u\right)+S\left(u, u, H x_{2 n-1}\right)\right]\right\} \\
= & \max \left\{S\left(x_{2 n-1}, x_{2 n-1}, u\right), S\left(x_{2 n-1}, x_{2 n-1}, x_{2 n}\right), S(u, u, H u),\right. \\
& \left.\frac{1}{2}\left[S\left(x_{2 n-1}, x_{2 n-1}, H u\right)+S\left(u, u, x_{2 n}\right)\right]\right\} \\
= & \max \left\{S\left(x_{2 n-1}, x_{2 n-1}, u\right), S\left(x_{2 n}, x_{2 n}, x_{2 n-1}\right), S(u, u, H u),\right. \\
& \left.\frac{1}{2}\left[S\left(x_{2 n-1}, x_{2 n-1}, H u\right)+S\left(x_{2 n}, x_{2 n}, u\right)\right]\right\}
\end{aligned}
$$

(by condition $\left(S M_{2}\right)$ )

$=\max \left\{S\left(x_{2 n-1}, x_{2 n-1}, u\right), S\left(x_{2 n}, x_{2 n}, x_{2 n-1}\right), S(u, u, H u)\right.$,

$$
\left.\frac{1}{2}\left[2 S\left(x_{2 n-1}, x_{2 n-1}, u\right)+S(u, u, H u)+S\left(x_{2 n}, x_{2 n}, u\right)\right]\right\}
$$

(by Lemma 1.8 and condition $\left(S M_{2}\right)$ ).

Using equations (3.26), (3.27), and (3.28) in (3.30), we obtain

$$
\begin{aligned}
\Theta\left(x_{2 n-1}, x_{2 n-1}, u\right) \leq & \max \left\{\frac{1}{4} S(u, u, H u), \frac{1}{4} S(u, u, H u), S(u, u, H u),\right. \\
& \left.\frac{1}{2}\left[2 \cdot \frac{1}{4} S(u, u, H u)+S(u, u, H u)+\frac{1}{4} S(u, u, H u)\right]\right\},
\end{aligned}
$$

that is,

$$
\Theta\left(x_{2 n-1}, x_{2 n-1}, u\right) \leq S(u, u, H u)
$$

and

$$
\begin{aligned}
\theta\left(x_{2 n-1}, x_{2 n-1}, u\right)= & \min \left\{S\left(x_{2 n-1}, x_{2 n-1}, F x_{2 n-1}\right), S\left(x_{2 n-1}, x_{2 n-1}, H x_{2 n-1}\right),\right. \\
& \left.S\left(x_{2 n-1}, x_{2 n-1}, H x_{2 n-1}\right), S\left(x_{2 n-1}, x_{2 n-1}, F u\right)\right\} \\
= & \min \left\{S\left(x_{2 n-1}, x_{2 n-1}, x_{2 n}\right), S\left(x_{2 n-1}, x_{2 n-1}, x_{2 n}\right)\right. \\
& \left.S\left(x_{2 n-1}, x_{2 n-1}, x_{2 n}\right), S\left(x_{2 n-1}, x_{2 n-1}, F u\right)\right\} .
\end{aligned}
$$

Now using equations (3.31) and (3.32) in (3.29), we obtain

$$
\begin{aligned}
\psi\left(S\left(x_{2 n}, x_{2 n}, H u\right)\right) \leq & \psi(S(u, u, H u))-\phi(S(u, u, H u)) \\
& +L_{1} \min \left\{S\left(x_{2 n-1}, x_{2 n-1}, x_{2 n}\right), S\left(x_{2 n-1}, x_{2 n-1}, x_{2 n}\right),\right. \\
& \left.S\left(x_{2 n-1}, x_{2 n-1}, x_{2 n}\right), S\left(x_{2 n-1}, x_{2 n-1}, F u\right)\right\} .
\end{aligned}
$$

Letting $n \rightarrow \infty$ in inequality (3.33), we obtain

$$
\psi(S(u, u, H u)) \leq \psi(S(u, u, H u))-\phi(S(u, u, H u))
$$


which is a contradiction unless $S(u, u, H u)=0$. Hence, we conclude that $u=H u$. This shows that $u$ is a fixed point of $H$. Note that

$$
\begin{aligned}
S(u, u, F u) & \leq 2 S(u, u, H u)+S(F u, F u, H u) \\
& =2 S(u, u, u)+S(H u, H u, F u) \quad \text { (by Lemma 1.8) } \\
& <S(u, u, F u),
\end{aligned}
$$

which is a contradiction. Hence $S(u, u, F u)=0$, that is, $u=F u$. Thus $u$ is a common fixed point of $F$ and $H$.

Now to show that the common fixed point of $F$ and $H$ is unique, suppose $v$ is another common fixed point of $F$ and $H$ such that $v=F v=H v$ with $v \neq u$. From (3.1), we have

$$
\begin{aligned}
\psi(S(u, u, v)) & =\psi(S(F u, F u, H v)) \\
& \leq \psi(\Theta(u, u, v))-\phi(\Theta(u, u, v))+L_{1} \theta(u, u, v) \\
& \leq \psi(S(u, u, v))-\phi(S(u, u, v)) \quad(\text { since } \theta(u, u, v)=0),
\end{aligned}
$$

which is a contradiction unless $S(u, u, v)=0$. Thus we conclude that $u=v$. This shows that the common fixed point of $F$ and $H$ is unique.

If $L_{1}=0$ in Theorem 3.1, then we obtain the following result.

Corollary 3.2 Let $(X, S)$ be a complete S-metric space and $F, H: X \rightarrow X$ be two self mappings satisfying the inequality

$$
\psi(S(F x, F y, H z)) \leq \psi(\Theta(x, y, z))-\phi(\Theta(x, y, z))
$$

for all $x, y, z \in X$, where $M, \psi$ and $\phi$ are defined as in Theorem 3.1. Then there exists a unique point $u \in X$ such that $u=F u=H u$.

Proof Follows from Theorem 3.1 by taking $L_{1}=0$.

Remark 3.3 Corollary 3.2 extends Theorem 2.1 of Doric [3] for a complete metric space to that in the setting of a complete $S$-metric space considered in this paper.

$$
\text { If } L_{1}=0 \text { and } F=H=\mathcal{T} \text { in Theorem 3.1, then we obtain the following result. }
$$

Corollary 3.4 Let $(X, S)$ be a complete $S$-metric space and $\mathcal{T}: X \rightarrow X$ be a self-mapping satisfying the inequality

$$
\psi(S(\mathcal{T} x, \mathcal{T} y, \mathcal{T} z)) \leq \psi(\Theta(x, y, z))-\phi(\Theta(x, y, z))
$$

for all $x, y, z \in X$, where

$$
\Theta(x, y, z)=\max \left\{S(x, y, z), S(y, y, \mathcal{T} x), S(z, z, \mathcal{T} z), \frac{1}{2}[S(y, y, \mathcal{T} z)+S(z, z, \mathcal{T} y)]\right\},
$$

and where $\psi$ and $\phi$ are defined as in Theorem 3.1. Then $\mathcal{T}$ has a unique fixed point in $X$. 
Proof Follows from Theorem 3.1 by taking $L_{1}=0$ and $F=H=\mathcal{T}$.

Remark 3.5 Corollary 3.4 extends Theorem 2.2 of Doric [3] for a complete metric space to that in the setting of a complete $S$-metric space considered in this paper.

$$
\begin{aligned}
& \text { If } L_{1}=0 \text { and } \\
& \qquad \begin{aligned}
\Theta(x, y, z)= & \max \{S(x, y, z), S(y, y, F x), S(z, z, H z), \\
& \left.\frac{1}{2}[S(y, y, H z)+S(z, z, H y)]\right\} \\
= & S(x, y, z)
\end{aligned}
\end{aligned}
$$

in Theorem 3.1, then we obtain the following result as a corollary.

Corollary 3.6 Let $(X, S)$ be a complete S-metric space and $F, H: X \rightarrow X$ be two selfmappings satisfying the inequality

$$
\psi(S(F x, F y, H z)) \leq \psi(S(x, y, z))-\phi(S(x, y, z))
$$

for all $x, y, z \in X$, where $\psi$ and $\phi$ are defined as in Theorem 3.1. Then there exists a unique point $u \in X$ such that $u=F u=H u$.

Remark 3.7 Corollary 3.6 extends result of Dutta and Choudhury [4, Theorem 2.1] for a metric space to an $S$-metric space for a pair of maps.

Remark 3.8 If

$$
\begin{aligned}
\Theta(x, y, z)= & \max \{S(x, y, z), S(y, y, F x), S(z, z, H z), \\
& \left.\frac{1}{2}[S(y, y, H z)+S(z, z, H y)]\right\} \\
= & S(x, y, z)
\end{aligned}
$$

and $F=H=\mathcal{T}$ in Theorem 3.1, then we obtain Theorem 2.2.

Remark 3.9 If

$$
\begin{aligned}
\Theta(x, y, z)= & \max \{S(x, y, z), S(y, y, F x), S(z, z, H z), \\
& \left.\frac{1}{2}[S(y, y, H z)+S(z, z, H y)]\right\} \\
= & S(x, y, z),
\end{aligned}
$$

$L_{1}=0, F=H=\mathcal{T}$, and $\psi$ as the identity map in Theorem 3.1, then we obtain Corollary 2.5. 
Remark 3.10 If we take

$$
\begin{aligned}
\Theta(x, y, z)= & \max \{S(x, y, z), S(y, y, F x), S(z, z, H z), \\
& \left.\frac{1}{2}[S(y, y, H z)+S(z, z, H y)]\right\} \\
= & S(x, y, z),
\end{aligned}
$$

$L_{1}=0$, and $F=H=\mathcal{T}$ in Theorem 3.1, then we obtain Corollary 2.3 of this paper.

\subsection{Illustrations}

Example 3.11 Let $X=[0,1]$. We define $S: X^{3} \rightarrow \mathbb{R}_{+}$by

$$
S(x, y, z)= \begin{cases}0 & \text { if } x=y=z \\ \max \{x, y, z\} & \text { if otherwise }\end{cases}
$$

for all $x, y, z \in X$. Then $(X, S)$ is a complete $S$-metric space. We define $F, H: X \rightarrow X$ and $\psi$, $\phi$ on $\mathbb{R}_{+}$by $F(x)=\frac{x}{2}, H(x)=\frac{x}{3}, \psi(t)=\frac{4}{3} t^{2}$ and $\phi(t)=\frac{1}{3} t^{2}$ for all $x \in X$ and $t \in \mathbb{R}_{+}$.

Without loss of generality, we assume that $x>y>z$. Then choosing $x=1, y=\frac{1}{2}$, and $z=\frac{1}{4}$, we have

$$
\begin{aligned}
& S(F x, F y, H z)=\max \left\{\frac{x}{2}, \frac{y}{2}, \frac{z}{3}\right\}=\frac{x}{2}, \\
& S(x, y, z)=\max \{x, y, z\}=x, \\
& S(z, z, H z)=\max \left\{z, z, \frac{z}{3}\right\}=z, \\
& S(y, y, F x)=\max \left\{y, y, \frac{x}{2}\right\}=y, \\
& S(y, y, H z)=\max \left\{y, y, \frac{z}{3}\right\}=y, \\
& S(z, z, F y)=\max \left\{z, z, \frac{y}{2}\right\}=z,
\end{aligned}
$$

and

$$
\Theta(x, y, z)=\max \left\{x, y, z, \frac{1}{2}[y+z]\right\}=x .
$$

Now, we consider

$$
\begin{aligned}
\psi(S(F x, F y, H z)) & =\frac{x^{2}}{3} \leq x^{2}=[\Theta(x, y, z)]^{2} \\
& =\frac{4}{3}[\Theta(x, y, z)]^{2}-\frac{1}{3}[\Theta(x, y, z)]^{2} \\
& =\psi(\Theta(x, y, z))-\phi(\Theta(x, y, z)),
\end{aligned}
$$


that is,

$$
\psi(S(F x, F y, H z)) \leq \psi(\Theta(x, y, z))-\phi(\Theta(x, y, z)) .
$$

Thus inequality (3.35) of Corollary 3.2 holds. Hence $F$ and $H$ satisfy all the hypothesis of Corollary 3.2 and 0 is the unique common fixed point of $F$ and $H$.

Example 3.12 Let $X=[0,1]$. We define $S: X^{3} \rightarrow \mathbb{R}_{+}$by

$$
S(x, y, z)= \begin{cases}0 & \text { if } x=y=z \\ \max \{x, y, z\} & \text { if otherwise }\end{cases}
$$

for all $x, y, z \in X$. Then $(X, S)$ is a complete $S$-metric space. We define $F, H: X \rightarrow X$ and $\psi$, $\phi$ on $\mathbb{R}_{+}$by $F(x)=\frac{x}{2}, H(x)=0, \psi(t)=2 t^{2}$ and $\phi(t)=t^{2}$ for all $x \in X$ and $t \in \mathbb{R}_{+}$.

Without loss of generality, we assume that $x>y>z$. Then

$$
S(F x, F y, H z)=\max \left\{\frac{x}{2}, \frac{y}{2}, 0\right\}=\frac{x}{2}
$$

and

$$
S(x, y, z)=\max \{x, y, z\}=x .
$$

Now, we consider

$$
\begin{aligned}
& \psi(S(F x, F y, H z))=2 \cdot \frac{x^{2}}{4}=\frac{x^{2}}{2}, \\
& \psi(S(x, y, z))=2 x^{2} \quad \text { and } \quad \phi(S(x, y, z))=x^{2} .
\end{aligned}
$$

Therefore, we have

$$
\psi(S(F x, F y, H z))=\frac{x^{2}}{2} \leq 2 x^{2}-x^{2}=x^{2}=\psi(S(x, y, z))-\phi(S(x, y, z)) .
$$

Thus inequality (3.36) of Corollary 3.6 holds. Hence $F$ and $H$ satisfy all the hypothesis of Corollary 3.6 and 0 is the unique common fixed point of $F$ and $H$.

Example 3.13 Let $X=[0,1]$ and let $S$ be the usual $S$-metric, that is, $S(x, y, z)=|x-z|+|y-z|$ for all $x, y, z \in X$ be the $S$-metric on $X$. Then $(X, S)$ is a complete $S$-metric space. We define $F, H: X \rightarrow X$ and $\psi, \phi$ on $\mathbb{R}_{+}$by $F(x)=x-x^{2}, H(x)=x, \psi(t)=t$ and $\phi(t)=\frac{t^{2}}{6}$ for all $x \in X$ and $t \in \mathbb{R}_{+}$. Without loss of generality, we assume that $x>y>z$. Then choosing $x=1, y=\frac{1}{2}$, and $z=\frac{1}{4}$, we have

$$
\begin{aligned}
S(F x, F y, H z) & =|F x-H z|+|F y-H z| \\
& =\left|\left(x-x^{2}\right)-z\right|+\left|\left(y-y^{2}\right)-z\right| \\
& =\left[\left(x-x^{2}\right)-z\right]+\left[\left(y-y^{2}\right)-z\right]
\end{aligned}
$$




$$
\begin{aligned}
& =[(x-z)+(y-z)]-\left(x^{2}+y^{2}\right) \\
& \leq[(x-z)+(y-z)]-(x-y)^{2} \\
& \leq[(x-z)+(y-z)]-\frac{1}{6}[(x-z)+(y-z)]^{2} \\
& =S(x, y, z)-\phi(S(x, y, z)) \\
& =\psi(S(x, y, z))-\phi(S(x, y, z)) .
\end{aligned}
$$

Thus inequality (3.36) of Corollary 3.6 holds. Hence $F$ and $H$ satisfy all the hypothesis of Corollary 3.6 and 0 is the unique common fixed point of $F$ and $H$.

\section{Applications}

Three decades ago, the rise of the character of cancer infections has critically long-drawnout. For every three persons, one of them had cancer attributable to infections. Simultaneously, the prevention of infection-connected cancers is shifting from a cancer control to infection control, for example, vaccination and the detection of infected persons. In livelihood of this modification, the usage of infection diffusion models has entered the field of infection-connected cancer. These simulations are valuable to realize the infection transmission procedures, to evaluate the important parameters that manage the extent of infection, and to describe the possible impact of different precautionary measures.

The dynamic equation of growth takes the from of a differential equation $\chi^{\prime}(t)=\chi(\chi(t))$ (see [8]). Later Yang and Zhang [9] introduced the growth equation of the form $\chi^{\prime}(t)=$ $\Phi(\chi(\chi(t)))$. The fractional differential formula in terms of Riemann-Liouville operator was given by Ibrahim [10] to study the existence and uniqueness of a solution, and the entropy solution was discussed by Ibrahim and Jalab in [11]. In this study, we shall use a new fractional calculus called conformable calculus (CC). In general, CC is introduced as follows:

Definition 4.1 (Conformable Differential Operator (CDO)) Let $\beta$ be a fractional power such that $\beta \in[0,1]$. CDO $\mathcal{D}^{\beta}$ is conformable if $\mathcal{D}^{0}$ is the identity operator and $\mathcal{D}^{1}$ is the classical differential operator. In particular, the operator

$$
\mathcal{D}^{\beta} \chi(t)=\left(t^{1-\beta}\right) \frac{d \chi(t)}{d t}=\left(t^{1-\beta}\right) \chi^{\prime}(t)
$$

is conformable if for a differentiable function $\chi(t)$,

$$
\mathcal{D}^{0} \chi(t)=\chi(t) \quad \text { and } \quad \mathcal{D}^{1} \chi(t)=\frac{d}{d t} \chi(t)=\chi^{\prime}(t) .
$$

Moreover, in the theory of control systems, a proportional-differential controller for controlling resultant $v$ at time $t$ with two tuning criteria has the form

$$
v(t)=v_{p} \Sigma(t)+v_{d} \frac{d}{d t} \Sigma(t)
$$

where $v_{p}$ is the proportional gain, $v_{d}$ is the derivative gain, and $\Sigma$ is the error between the state variable and the process variable. Based on (4.1), Anderson and Ulness [12] introduced the following definition. 
Definition 4.2 For two continuous functions $v_{0}, v_{1}:[0,1] \times \mathbb{R} \rightarrow(0, \infty)$, define

$$
\mathcal{D}^{\beta} \chi(t)=v_{1}(\beta, t) \chi(t)+v_{0}(\beta, t) \chi^{\prime}(t)
$$

such that $v_{1}(\beta, t) \neq-v_{0}(\beta, t)$,

$$
\lim _{\beta \rightarrow 0} v_{1}(\beta, t)=1, \quad \lim _{\beta \rightarrow 1} v_{1}(\beta, t)=0, \quad v_{1}(\beta, t) \neq 0, \forall t, \beta \in(0,1)
$$

and

$$
\lim _{\beta \rightarrow 0} v_{0}(\beta, t)=0, \quad \lim _{\beta \rightarrow 1} v_{0}(\beta, t)=1, \quad v_{0}(\beta, t) \neq 0, \forall t, \beta \in(0,1) .
$$

Note that CC satisfies the integral equation

$$
\int \mathcal{D}^{\beta} \chi(t) d_{\beta} t=\chi(t)+c e_{0}\left(t, t_{0}\right)
$$

where $c \in \mathbb{R}, d_{\beta} t=\frac{d t}{v_{0}(t)}, \nu \neq 0$ and $e_{0}(t, \tau)=\exp \left(-\int_{\tau}^{t} \frac{\nu_{1}(\beta, \zeta)}{\left.\nu_{0}(\beta, \zeta)\right)} d \varsigma\right)$. The above definition was extended to the complex domain in [13].

In this application, we aim to employ Theorem 2.2 to establish the existence and uniqueness of a solution of the conformable equation

$$
\begin{aligned}
\mathcal{D}^{\beta} \chi(t) & =v_{1}(\beta, t) \chi(t)+v_{0}(\beta, t) \chi^{\prime}(t) \\
& =v_{1}(\beta, t) \chi(t)+v_{0}(\beta, t) \Phi(\chi(\chi(t)),
\end{aligned}
$$

where $\chi, \Phi:[0, \infty) \rightarrow[0, \infty)$ are continuous nondecreasing functions such that $\chi(t)>0$ for all $t \in(0, \infty)$ and $\Phi(0)=\chi(0)=0$. It is clear that, when $v \rightarrow 1$, we obtain Yang and Zhang formula [9], and when $\Phi(y)=y$, we get Eder formula [8]. In our discussion, we shall select one of the following formulas of $v_{1}$ and $v_{0}$ :

$$
\begin{array}{ll}
v_{1}(\beta, t)=(1-\beta) t^{\beta}, & v_{0}(\beta, t)=\beta t^{1-\beta}, \quad t \in(0, \infty), \\
v_{1}(\beta, t)=(1-\beta)|t|^{\beta}, & v_{0}(\beta, t)=\beta|t|^{1-\beta}, \\
v_{1}(\beta, t)=\cos \left(\frac{\beta \pi}{2}\right) t^{\beta}, & v_{0}(\beta, t)=\sin \frac{\beta \pi}{2} t^{1-\beta}, \quad t \in(0, \infty),
\end{array}
$$

or

$$
v_{1}(\beta, t)=\cos \left(\frac{\beta \pi}{2}\right)|t|^{\beta}, \quad v_{0}(\beta, t)=\sin \frac{\beta \pi}{2}|t|^{1-\beta}, \quad t \in \mathbb{R} \backslash\{0\} .
$$

Furthermore, constant functions can be realized by using the gamma function as follows:

$$
v_{1}(\beta, t)=\frac{(1-\beta)}{\Gamma(\beta+1)}, \quad v_{0}(\beta, t)=\frac{\beta}{\Gamma(\beta+1)} .
$$

Denoting $X=\mathbb{R}$ and in view of (4.3) and (4.4), we define an operator $Q ; X \rightarrow X$ as follows:

$$
(Q \chi)(t)=\int_{0}^{t}\left(v_{1}(\beta, t) \chi(t)+v_{0}(\beta, t) \Phi(\chi(\chi(t))) d_{\beta} \varsigma+c e_{0}\left(t, t_{0}\right) .\right.
$$


Since $\chi \in X, Q$ is a self-mapping. In addition, define a function $\mathfrak{S}: X^{3} \rightarrow \mathbb{R}^{+}$by

$$
\mathfrak{S}\left(\chi_{1}, \chi_{2}, \chi_{3}\right)=\max \left\{\left|\chi_{l}-\chi_{J}\right|: \imath, J=1,2,3, \imath \neq J\right\}
$$

where $\chi_{1}(t)=\chi(t), \chi_{2}(t)=\chi(\chi(t))$ and $\chi_{3}(t)=\chi(\chi(\chi(t))):=\Phi(\chi(\chi(t)))$. Obviously, $\mathfrak{S}\left(\chi_{1}, \chi_{2}, \chi_{3}\right)=0$ for $\chi_{1}=\chi_{2}=\chi_{3}$; also, we have

$$
\begin{aligned}
& \mathfrak{S}\left(\chi_{1}, \chi_{1}, \chi_{i}\right)+\mathfrak{S}\left(\chi_{2}, \chi_{2}, \chi_{j}\right)+\mathfrak{S}\left(\chi_{3}, \chi_{3}, \chi_{k}\right) \\
&=\max _{i=2,3}\left\{\left|\chi_{1}-\chi_{i}\right|\right\}+\max _{j=1,3}\left\{\left|\chi_{2}-\chi_{j}\right|\right\}+\max _{k=1,2}\left\{\left|\chi_{3}-\chi_{k}\right|\right\} \\
&=\max \left\{\left|\chi_{1}-\chi_{2}\right|, \mid \chi_{1}-\chi_{3}\right\}+\max \left\{\left|\chi_{2}-\chi_{1}\right|, \mid \chi_{2}-\chi_{3}\right\} \\
& \quad+\max \left\{\left|\chi_{3}-\chi_{1}\right|,\left|\chi_{3}-\chi_{2}\right|\right\} \\
&= 2 \max \left\{\left|\chi_{1}-\chi_{2}\right|,\left|\chi_{2}-\chi_{3}\right|,\left|\chi_{3}-\chi_{1}\right|\right\} \\
&> \max \left\{\left|\chi_{1}-\chi_{2}\right|,\left|\chi_{2}-\chi_{3}\right|,\left|\chi_{3}-\chi_{1}\right|\right\} \\
&= \max \left\{\left|\chi_{l}-\chi_{J}\right|: l, J=1,2,3, l \neq J\right\} \\
&= \mathfrak{S}\left(\chi_{1}, \chi_{2}, \chi_{3}\right) .
\end{aligned}
$$

Hence, the metric $\mathfrak{S}\left(\chi_{1}, \chi_{2}, \chi_{3}\right)$ is an S-metric on the set $X$.

Theorem 4.3 Consider the conformable equation (4.4). If

$$
\mid \Phi(\chi(\chi(t))-\Phi(\eta(\eta(t))|<\ell| \chi(t)-\eta(t) \mid
$$

for some positive constant $\ell<\frac{1-(1-\beta) \mathfrak{T}^{\beta}}{\beta \mathfrak{T}^{1-\beta}}, \mathfrak{T}<\infty$, then $Q$ has a unique fixed point in $X$.

Proof Let the functions $v_{0}$ and $v_{1}$ be given by

$$
v_{1}(\beta, t)=(1-\beta) t^{\beta}, \quad v_{0}(\beta, t)=\beta t^{1-\beta}, \quad t \in(0, \mathfrak{T}), \mathfrak{T}<\infty .
$$

Then, by utilizing the inequality of $\ell$, we have

$$
\begin{aligned}
\mathfrak{S}( & \left.Q \chi_{1}(t), Q \chi_{2}(t), Q \chi_{3}(t)\right) \\
= & \max \left\{\left|Q \chi_{l}(t)-Q \chi_{J}(t)\right|: l, J=1,2,3, l \neq J\right\} \\
\leq & \max \left\{\mid v_{1}(\beta, t) \chi_{l}(t)+v_{0}(\beta, t) \Phi\left(\chi_{l}\left(\chi_{l}(t)\right)\right.\right. \\
& -\left(v_{1}(\beta, t) \chi_{J}(t)+v_{0}(\beta, t) \Phi\left(\chi_{J}\left(\chi_{J}(t)\right)\right) \mid \frac{\mathfrak{T}^{\beta}}{\beta^{2}}: l, J=1,2,3, l \neq J\right\} \\
\leq & \max \left\{v_{1}(\beta, t)\left|\chi_{l}-\chi_{J}\right| \frac{\mathfrak{T}^{\beta}}{\beta^{2}}+v_{0}(\beta, t) \ell\left|\chi_{l}-\chi_{J}\right| \frac{\mathfrak{T}^{\beta}}{\beta^{2}}: l, J=1,2,3, l \neq J\right\} \\
= & \max \left\{\left[(1-\beta) \mathfrak{T}^{\beta}+\beta \mathfrak{T}^{1-\beta} \ell\right] \frac{\mathfrak{T}^{\beta}}{\beta^{2}}\left|\chi_{l}-\chi_{J}\right|: l, J=1,2,3, l \neq J\right\} \\
:= & r \mathfrak{S}\left(\chi_{1}, \chi_{2}, \chi_{3}\right) .
\end{aligned}
$$


Hence, $Q$ is bounded in the unit ball $B_{r}$ of radius $0<r<1$. We proceed to discover more properties of the operator $Q$. Let $t, \tau \in(0, \mathfrak{T})$ be such that $t>\tau$. Then $\chi(t)>\chi(\tau)$ (increasing function). A computation yields that

$$
\begin{aligned}
\mathfrak{S} & \left(Q \chi_{1}(t), Q \chi_{2}(t), Q \chi_{3}(t)-\left(Q \chi_{1}(\tau), Q \chi_{2}(\tau), Q \chi_{3}(\tau)\right)\right. \\
& =\mathfrak{S}\left(Q\left(\chi_{1}(t)-\chi_{1}(\tau)\right), Q\left(\chi_{2}(t)-\chi_{2}(\tau)\right), Q\left(\chi_{3}(t)-\chi_{3}(\tau)\right)\right) \\
& =\mathfrak{S}\left(Q \chi_{1}(t-\tau), Q \chi_{2}(t-\tau), Q \chi_{3}(t-\tau)\right) \\
& \leq \mathfrak{S}\left(Q \chi_{1}(t), Q \chi_{2}(t), Q \chi_{3}(t)\right) \\
& \leq r \mathfrak{S}\left(\chi_{1}, \chi_{2}, \chi_{3}\right) .
\end{aligned}
$$

Thus, $Q$ is equicontinuous on $B_{r}$. Moreover, by letting $\chi_{l}(t)-\eta_{l}(t)=\xi_{l}(t), l=1,2,3$, we attain that

$$
\begin{aligned}
\mathfrak{S} & \left(Q\left(\chi_{1}(t)-\eta_{1}(t)\right), Q\left(\chi_{2}(t)-\eta_{2}(t)\right), Q\left(\chi_{3}(t)-\eta_{3}(t)\right)\right) \\
= & \mathfrak{S}\left(Q\left(\xi_{1}(t)\right), Q\left(\xi_{2}(t)\right), Q\left(\xi_{3}(t)\right)\right) \\
\leq & \max \left\{\mid v_{1}(\beta, t) \xi_{l}(t)+v_{0}(\beta, t) \Phi\left(\xi_{l}\left(\chi_{l}(t)\right)\right.\right. \\
& -\left(v_{1}(\beta, t) \xi_{J}(t)+v_{0}(\beta, t) \Phi\left(\xi_{J}\left(\xi_{J}(t)\right)\right) \mid \frac{\mathfrak{T}^{\beta}}{\beta^{2}}: l, J=1,2,3, l \neq J\right\} \\
\leq & \max \left\{v_{1}(\beta, t)\left|\xi_{l}-\xi_{J}\right| \frac{\mathfrak{T}^{\beta}}{\beta^{2}}+v_{0}(\beta, t) \ell\left|\xi_{l}-\xi_{J}\right| \frac{\mathfrak{T}^{\beta}}{\beta^{2}}: l, J=1,2,3, l \neq J\right\} \\
\leq & \max \left\{(1-\beta) \mathfrak{T}^{\beta}\left|\xi_{l}-\xi_{J}\right| \frac{\mathfrak{T}^{\beta}}{\beta^{2}}+\beta \mathfrak{T}^{1-\beta} \ell\left|\xi_{l}-\xi_{J}\right| \frac{\mathfrak{T}^{\beta}}{\beta^{2}}: l, J=1,2,3, l \neq J\right\} \\
= & \max \left\{\left[(1-\beta) \mathfrak{T}^{\beta}+\beta \mathfrak{T}^{1-\beta}(\beta, t) \ell\right] \frac{\mathfrak{T}^{\beta}}{\beta^{2}}\left|\xi_{l}-\xi_{J}\right|: l, J=1,2,3, l \neq J\right\} \\
\leq & r \mathfrak{S}\left(\xi_{1}, \xi_{2}, \xi_{3}\right) \leq r \mathfrak{S}\left(\chi_{1}, \chi_{2}, \chi_{3}\right) .
\end{aligned}
$$

Therefore, the operator $Q$ is continuous in $B_{r}$. This implies that $Q$ has a fixed point $Q \chi=\chi$.

Next we aim to check inequality (2.1). Suppose that there are two continuous and nondecreasing functions $\psi, \phi:[0, \infty) \rightarrow[0, \infty)$ with the properties: $\psi(t), \phi(t)>0$ for $t>0$ and $\psi(t)=\phi(t)=0$ if and only if $t=0$. Now, by putting $\psi(\rho)=\rho / r$ and $\phi(\rho)=\frac{\rho(1-r)}{r}$, from the boundedness of $Q$ and (4.9), we conclude that

$$
\begin{aligned}
& \psi\left(\mathfrak{S} Q\left(\chi_{1}, \chi_{1}, \chi_{i}\right)\right) \\
& =\frac{\mathfrak{S} Q\left(\chi_{1}, \chi_{1}, \chi_{i}\right)}{r} \leq \mathfrak{S}\left(\chi_{1}, \chi_{2}, \chi_{3}\right) \\
& \leq \mathfrak{S}\left(\chi_{1}, \chi_{1}, \chi_{i}\right)+\mathfrak{S}\left(\chi_{2}, \chi_{2}, \chi_{j}\right)+\mathfrak{S}\left(\chi_{3}, \chi_{3}, \chi_{k}\right) \\
& =\psi\left(\mathfrak{S}\left(\chi_{1}, \chi_{1}, \chi_{i}\right)\right)-\phi\left(\mathfrak{S}\left(\chi_{1}, \chi_{1}, \chi_{i}\right)\right)+\mathfrak{S}\left(\chi_{2}, \chi_{2}, \chi_{j}\right)+\mathfrak{S}\left(\chi_{3}, \chi_{3}, \chi_{k}\right) \\
& \leq \psi\left(\mathfrak{S}\left(\chi_{1}, \chi_{1}, \chi_{i}\right)\right)-\phi\left(\mathfrak{S}\left(\chi_{1}, \chi_{1}, \chi_{i}\right)\right) \\
& +\min \left\{\mathfrak{S}\left(\chi_{2}, \chi_{2}, Q \chi_{2}\right), \mathfrak{S}\left(\chi_{2}, \chi_{2}, Q \chi_{1}\right), \mathfrak{S}\left(\chi_{1}, \chi_{1}, Q \chi_{1}\right), \mathfrak{S}\left(\chi_{1}, \chi_{1}, Q \chi_{2}\right)\right\} .
\end{aligned}
$$


Hence, with $L_{1}=1$ we get inequality (2.1). Then, in view of Theorem 2.2 , we conclude that $Q$ has a unique fixed point lying in $B_{r}, r<1$.

\subsection{Numerical example}

Consider the following data: $t \in(0,1], \beta=0.5, \Phi(w)=0.5 w$, and the conformable iterative equation

$$
\begin{aligned}
\mathcal{D}^{0.5} \chi(t) & =v_{1}(\beta, t) \chi(t)+v_{0}(\beta, t) \chi^{\prime}(t) \\
& =v_{1}(0.5, t) \chi(t)+0.5 v_{0}(0.5, t)(\chi(\chi(t)),
\end{aligned}
$$

It is easy to compute $\ell=0.5<1$. Then equation (4.10) has a unique solution (see Theorem 4.3) in the unit ball $B_{r}$, where $r=\left[(1-\beta) \mathfrak{T}^{\beta}+\beta \mathfrak{T}^{1-\beta} \ell\right]=0.75$. By using this method, one can generalize to other types of differential equations to improve the solutions.

\section{Conclusion}

In this paper, we have defined $(\psi-\phi)$-almost weakly contractive maps in $S$-metric spaces and established several unique fixed point and unique common fixed point theorems in the framework of complete $S$-metric spaces. Also we have given some examples in support of our results. They extend, generalize, and unify the corresponding results of [3-5], and many others from the existing literature to those in the setting of complete $S$-metric spaces considered in this paper.

\section{Acknowledgements}

The authors would like to thank the Editor and Reviewers in helping improve the manuscript.

\section{Funding}

This research is funded by the Foundation for Science and Technology Development of Ton Duc Thang University (FOSTECT), website http://fostect.tdtu.edu.vn, under Grant FOSTECT.2019.14.

Availability of data and materials

Not applicable.

Competing interests

The authors declare that they have no competing interests.

Authors' contributions

The authors declare that the study was realized in collaboration with the same responsibility. All authors read and approved the final manuscript. All authors contributed equally to the writing of this paper.

\section{Author details}

${ }^{1}$ Faculty of Mathematics and Statistics, Ton Duc Thang University, Ho Chi Minh City, Vietnam. ${ }^{2}$ Department of Mathematics, Govt. Kaktiya P. G. College Jagdalpur, Jagdalpur, India. ${ }^{3}$ Informetrics Research Group, Ton Duc Thang University, Ho Chi Minh City, Vietnam.

\section{Publisher's Note}

Springer Nature remains neutral with regard to jurisdictional claims in published maps and institutional affiliations.

Received: 18 January 2020 Accepted: 16 April 2020 Published online: 13 May 2020

\section{References}

1. Alber, Y.I., Guerre-Delabrieer, S.: Principles of weakly contractive maps in Hilbert spaces. In: Gohberg, I., Lyubich, Y. (eds.) New Results in Operator Theory, Advances and Appl, vol. 98, pp. 7-22. Birkhäuser, Basel (1997)

2. Babu, G.V.R., Babu, D.R., Rao, K.N., Kumar, B.V.S.: Fixed points of $(\psi, \varphi)$-almost weakly contractive maps in G-metric spaces. Appl. Math. E-Notes 14, 69-85 (2014)

3. Doric, D.: Common fixed point for generalized $(\psi, \varphi)$-weak contractions. Appl. Math. Lett. 22, 1896-1900 (2009)

4. Dutta, P.N., Choudhury, B.S.: A generalisation of contraction principle in metric spaces. Fixed Point Theory Appl. 2008, Article ID 406368 (2008) 
5. Rhoades, B.E.: Some theorems on weakly contractive maps. Nonlinear Anal. TMA 47(4), 2683-2693 (2001)

6. Sedghi, S., Shobe, N., Aliouche, A.: A generalization of fixed point theorems in S-metric spaces. Mat. Vesn. 64(3), 258-266 (2012)

7. Sedghi, S., Dung, N.V.: Fixed point theorems on S-metric spaces. Mat. Vesn. 66(1), 113-124 (2014)

8. Eder, E.: The functional differential equation $x^{\prime}(t)=x(x(t))$. J. Differ. Equ. 54, 390-400 (1984)

9. Yang, D., Zhang, W.: Solutions of equivariance for iterative differential equations. Appl. Math. Lett. 17(7), 759-765 (2004)

10. Ibrahim, R.W.: Existence of iterative Cauchy fractional differential equation. J. Math. 2013, 1-8 (2013)

11. Ibrahim, R.W., Hamid, J.: Existence of Ulam stability for iterative fractional differential equations based on fractional entropy. Entropy 17(5), 3172-3181 (2015)

12. Anderson, D.R., Ulness, D.J.: Newly defined conformable derivatives. Adv. Dyn. Syst. Appl. 10(2), 109-137 (2015)

13. Ibrahim, R.W., Jahangiri, J.M.: Conformable differential operator generalizes the Briot-Bouquet differential equation in a complex domain. AIMS Math. 4(6), 1582-1595 (2019)

\section{Submit your manuscript to a SpringerOpen ${ }^{\circ}$} journal and benefit from:

- Convenient online submission

Rigorous peer review

- Open access: articles freely available online

- High visibility within the field

- Retaining the copyright to your article

Submit your next manuscript at $\gg$ springeropen.com 